partially detached bone, and these no doubt materially helped forward the satisfactory result.

For some months after the injury well-marked pulsation of the brain continued and the bony edges of the defect could be easily recognized; but when the patient was examined after a lapse of sixteen months a thick cicatrix had developed, the whole margin of the opening was well covered with firm tissue, and only a very feeble pulsation could be detected. I have practised this simple method in many cases of head injury with equally good results.

In concluding these remarks I desire to accentuate the value of utilizing scales and small fragments of bone in cases of extensive fracture of the skull, for the purpose of securing the development of a sound and thick cicatrix, a result which may be anticipated with confidence provided the wound is rendered thoroughly aseptic at the time of the operation, and the general condition of the patient continues favourable for gradual recovery.

The performance of secondary osteoplastic operations for the closure of large openings ought to be limited to cases in which persistent headache and other severe head symptoms are present, and there is evidence that these disorders can be attributed to the injury. Under these circumstances surgical interference may be undertaken for the relief of suffering which appears to be caused by some local irritation involving the cerebral cortex, and associated with cicatricial thickening and adhesion of the membranes.

\section{CASE OF SUBCUTANEOUS INJURY OF THE} PANCREAS : OPERATION : RECOVERY.

By GEORGE HEBB COWEN, M.S.Lond., F.R.C.S.ENG., ASSISTANT SURGEON, ROYAL SOUTH HANTS AND SOUTHAMPTON HOSPITAL ; CONSULTING SURGEON, SOUTHAMPTON INCORPORATION INFIRMARY.

CASES of subcutaneous injury to the pancreas without involvement of other organs must be extremely rare; in fact, it is difficult to concei ve how such an injury can take place. Miknlicz (Moynihan's Abdominal Operations) had collected up to July, 1903, twenty-four recorded cases; of these thirteen were not operated upon, and all died; and of eleven which were operated upon, seven recovered. Some of the operations were late, and consisted of evacuating disintegrating blood clot and providing drainage.

J. H., a man aged 38, was admitted to the Roval South Hants and Southampton Hóspital on October 10th, 1906.

History.

On October 6th he was walking home after dark on a road skirting the river Itchen, which was guarded by a wooden ralling. He had taken a fair amount of liquor that night, and, his foot striking some obstruction, he fell over the rail into the river, hitting his abdomen as he did so. He was rescued and taken home, but remembered nothing until the next morning. He then had great pain in the stomach and back, shooting up into the chest. The pain came on worse in paroxysms, and was accompanied by severe vomiting, the vomit being of a greenish colour. He remained in much the same condition till October 10 th, when he became much worse, the pain having spread all over the abdomen. He sent for Dr. Jackson of Eastleigh, who at once advised his removal to hospital. The day previously he had taken three doses of salts, but there had been no bowel action, though a little flatus had been passed.

\section{State on Admission.}

He looked extremely ill and was very pallid. The pulse was 100 and very small; temperature, $98.8^{\circ} \mathrm{F}$. The abdomen was uniformly distended but not greatly so, and there was much rigidity; there was a little dullness in both flanks and in the hypogastrium, the rest of the abdomen was tympanitic. Liver dullness was present. There was tenderness all over. Rectal examination was negative. The examination of the chest yielded notbing of moment. Urine drawn off by catheter was healthy, a small quantity only being obtained. He complained of great abdominal pain and of much thirst. The case was regarded as one of peritonitis and exploration decided upon; it was carried out shortly after admission.

Operation.

The abdomen was opened a little to the left of the middle line between the umbilicus and pubes by an incision about 3 in. in length. Liquid blood began to flow out from all directions as soon as the peritoneum was opened. The incision was enlarged upwards and downwards, and th lower viscera explored, no injury or disease being detected; there was much clot in the pelvis. The hand, passed upwards, came across a bulging, semi-fluid swelling above the navel ; an incision was therefore made above the umbilicus, about 4 in. in length, when blood was seen coming through 8 hole in the omentum, between the stomach and transverse colon. The two cuts were now joined, and it became apparent that the swelling was the lesser sac filled with clot and fluid blood. The opening in the omentum was enlarged by tearing and the clot removed by handfuls. Dark liquid blood continually welled up from the region of the pancreas, obscuring everything so that a view of the source of the flow was impossible. It was obvious that the only thing to be done was to trust to gauze pressure, and accordingly gauze was packed firmly gainst the pancre back of the lesser sac, quite through and through silkworm-gut stitches and several pints through and through silkworm-gut stitches, and several pints
of saline fluid poured into the general cavity. The end of the gauze was brought out of the wound on a level with the hole in the gastro-colic omentum. The operation had to be completed as rapidly as possible owing to the collapsed condition of the patient.

The next day, October 11 th, the man had recovered to some extent from the shock. Temperature was $99.2^{\circ} \mathrm{F}$.; pulse about 100, and extremely feeble. He vomited on and off all day. Saline fluid was given by the bowel. An enema in the evening brought away a fair amount of flatus. There had been oozing from the wound; the dressin

graze plugging being left untouched.

On October 12th he was distinctly better, and was able to take fluid nourishment. Calomel was given with good result. Dressing changed. Discharge thinner.

October 13th. Still improving. Pulse 86. The gauze plug was becoming lonsened by the blood-stained discharge, and part of it was removed by gentle traction.

October 15th. The rest of the gauze packing was removed quite easily. There was much discharge of blood-stained fluid.

The wound healed up fairly well, except for the sinus where. the plug had been. and from this was discharged daily 6 to $8 \mathrm{oz}$. of clear fluid. This fluid was most irritating to the skin, causing a very painful dermatitis wherever it came in contact with it. The skin condition was relieved by a catheter conveying the fluid from the sinus to a bottle. A specimen of the fluid was sent up to the Clinical Research Association, and was reported to be pancreatic.

He was discharged well on December 12th, fitted with an abdominal belt, to which was fixed a celluloid flask with a small rabber tube leading into it. the other end of which. inserted into the sinus, conveyed the juice awsy without contact with the skin.

When seen last, in March, 1907, he was doing his work, the sinus still discharging 3 or $4 \mathrm{oz}$. of fluid a day; belt and apparatus as before.

On one occasion (January, 1907) the sinus had closed, as he had left out the tube for some days. He came up complaining of pain in the back. A small nick was made at the site of the sinus, and several ounces of fluid let out, with im mediate relief; the tube was then put back.

Before operation no diagnosis was made, in fact the condition was extremely puzzling. There was the history' of injury with much pallor and signs of commencing peritonitis, the patient having become much worse the day of admission. There was no bruising to be found anywhere. Ruptured bladder was thought of, as there had been some difficalty of micturition with frequency. On opening the abdomen the appearance was just like that of a ruptared ectopic gestation. When the abdomen was completely laid open by joining the two incisions, a rapid examination of liver, spleen, mesentery, etc., disclosed no apparent injury, the trouble being confined to the lesser sac.

There is no doubt that laceration of the pancreas had taken place, as was proved afterwards by the discharge of pancreatic juice. An irregular mass could be felt at the back of the cavity; but no view could be obtained, owing to the continual flow of blood.

Evidently bleeding at first was confined to the lesser sac, and the paroxysmal increase in the pain the patient suffered the first day or two was probably due to suc. cessive haemorrhages into the distended cavity. When the man became so much worse on October 10th, the extravasation had doubtless burst through the omentum between the stomach and colon, involving the general cavity of the peritoneum.

Many cases of pancreatic cyst so called have followed injuries to the upper part of the abdomen, and are probably caused by blood extravasations into the lesser sac with a wound of the pancreas allowing the escape of a certain amount of pancreatic secretion. In this case it is 
quite possible that if the secondary giving way of the gastro-colic omentum had not occurred, a pseudo-pancreatic cyst would have been produced hat the patient survived without operation.

\section{RETROPERITONEAL SARCOMA OF DOUGLAS'S POUCH.}

BY

C. FERRIER WALTERS, and I. WALKER HALL, F.R.C.S.,

SURGICAL REGISTRAR, ROYAL INFIRMARY, BRISTOL. M.D.

PATHOLOGIST TO THE ROYA INFIRMARY, BRISTOL PROFESSOR OF PATHOLOGY, UA IVERSITY COLLEGE, BRISTO L.

Retroperitoneal growths have assumed considerable importance within recent years, and a large number of benign and malignant growths have been recorded. An admirable summary of these is given by Steele. ${ }^{1}$

Of growths arising in the retroperitoneal tissue of Douglas's pouch, only 7 cases appear to be reported in the literature at hand. The paucity of this record justifies the mention of the following instance.

A married woman, aged 35, was admitted to the Bristol Royal Infirmary in October, 1906, for the treatment of an abdominal swelling. Prior to 1904 her health had been good. In 1904, Dr. James Swain operated upon her for an attack of cholelithiasis. From March, 1905, she complained of "dragging" pains in the pelvis and thighe, and pain, located at the lower part of the abdomen, during micturition. Wasting had been gradual and continuous.

On admission, micturition was frequent, but painless. Menstruation regular. The "period" which took place two weeks before admission was, however, accompanied by a certain amount of pain.

On examination, the swelling was found to be located in the pelvis. It was about the size of a fetal head, was situated between the uterus and rectum, and was firm, and nodular in consistence. The uterus was disolaced upwards and forwards, and the os uteri could only be felt at the level of, and immediately posterior to, the symphysis pubis. The new growth appeared to be connected with the posterior wall of the upper portion of the cervix uteri, and with the anterior wall of the rectum. The body of the uterus, the ovaries, and other uterine appendages were not involved. The superficial veins of the thighs and legs were enlarged; the abdominal veins were unaffected.

The differential diagnosis compelled the consideration of fibroids, uterine and rectal growths, retroperitoneal and intraligamentous tumours, dermoids, and myeloid and periosteal new formations. Dr. Swain sent the case in as a "fibroid," with the alternative diagnosis of malignant pelvic growth.

Operation.-The usual median subumbilicai incision exposed a vascular, soft growth, covered by a thin, shiny capsule, which filled the posterior half of the true pelvis, being apparently attached to the cervix uteri anteriorly, and to the rectum posteriorly. When the peritoneum was cut through, a friable haemorrhagic mass was revealed. The attempt to remove this tissue occasioned such free haemorrhage that it was a matter of necessity to treat the haemorihage only, and desist from further removal of the growth. Death occurred fortp-eight hours later.

At the autopsy it was found that the organs were generally healthy, and there were no signs of postoperative haemorrhage. The tumour arose in the retroperitoneal tissue on the left side of Douglas's pouch. Here there was a mass, about the size and shape of a fivemonths placenta, cream-white in colour, fairly firm in consistence, separated from the utero-vaginal and rectal tissues by a thick, well-defined fibrous capsule, from which radiating trabeculae passed into the tumour, and situated chiefly adjacent to the vaginal wall but extending also into subperineal and perirectal tissues. This part of the growth lay with its long axis parallel to that of the aterus, and measured $4 \frac{1}{2}$ by 3 in. From this area of origin extension had taken place across the middle line, a small proportion of the mass reaching the right side of the pouch. An extension upwards had also occurred, but in this direction the tumour began to lose its solid characters, and exhibited haemorrhages, degenerations, and cystic collections. Superiorly it had lost the capsular tissue which was so apparent at its lower margins, and was covered only by the peritoneum itself.

There were no metastases; the pelvic bones were normal. When the uterus was bisected it was seen that the body of the uterus was normal. Its internal surface presented a blnod-stained area, which microscopical examination showed to consist of haemorrhage and oedema only. The tumour was adherent to the posterior lip of the cervix uteri, and to the entire length of the posterior fornix of the vagina. It then occupied the whole of the subperineal tissue, and became adherent to the anterior wall of the rectum by perirectal tissue. In these areas a distinct capsule was apparent; this capsule became thinner as it passed upwards, the thin peritoneal covering being alone present at the advancing edge. The bladder was quite unaffected.

Microscopically, the capsule was composed of vaseular fibrous tissue. At its inner border there were evidences of a slight invasion of tumour cells. The coats of the rectum were quite normal. The vaginal wall was not infiltrated. The tumour itself was composed of sarcomatous cells. These varied in type, large and small round cells and small spindle cells being present. Mitotic figures were easily demonstrable. The adipose tissue of the part was represented by masses of fat crystals and degenerating fat cells, infiltrated slightly by sarcoma cells. There were no evidences of dermoid or implantation structures, nor of any cells of a glandular type. From the characters of its cells, its blood vessels, and its degenerations, the tumour was considered as a mixed cell sarcoma.

Before discussing the prognosis, treatment, and etiology of these growths, it may be well to shortly recount the cases previously recorded in the literature to which access is possible.

Boy, aged 9. The clinical diagnosis was lympho-sarcoma of the pelvis. For eight months a swelling had been noticed in the abdomen; fourteen days before death alteration in hearing and speech and loss of intelligence were observed. Post mortem, there was slight oedema of the brain, pus in both tympanic cavitie?, pulmonary congestion, bronchial catarrh, and dislension of the bladder and renal pelvis. Between the bladder and rectum there was a nodular tumour the eize of a fetal head, rising slightly out of the true pelvie. A most careful examination of the tumour was made, but no connexion with any pelvic organ could be ascertained. On section it appeared dark red in colour, streaks of connective tissue could be seen running through it, and in places it was yellow, apparently fatty. The mass was completely enclosed in a thick capsule.

Microscopically, the tumour was composed throughout of a vascular connective-tissue stroma, forming irregular meshes, and contained spindle and round sarcoma cells, the former predominating; there were areas of necrosis and degeneration.

Spindle-celled Sarcoma (McLachlan ${ }^{3}$ ).

An elderly unmarried woman complained of anorexia sleeplessness, malaise, and a feeling of fullness in the abdomen, with slight pain; a definite tumour was found, which steadily increased in size, and eventually reached the dimensions of an eight months pregnancy. It was dull on percussion, and the superficial veins of the abdomen were enlarged, especially on the left side. The patient died suddenly in an attack of dyspnoea. Post mortem the examination was limited to the abdomen. A tumour, arising from the pelvis, was found to be adheren't to the whole anterior surface of the sacrum and to the uterus and tubes, the ovaries being free. Microscopically, it was a spindle-celled sarcoma, with many giant cells and large chondrifying areas.

Cystoma of Congenital Origin (Krönig ${ }^{4}$ ).

This was a retroperitoneal growtb, arising between the uterus and rectum, and reaching to the umbilicus; it was diagnosed as an intraligamentous cyst. Laparotomy showed that it was not attached to the uterine appendages, but that it was intimately connected with the pelvic floor and the posterior wall of the uterus. During removal it had to be separated from dense adhesions. The broad ligaments were not involved, but the peritoneum of the posterior pelvic wall and the right side of the sigmoid was pushed upwards by the growth. 